\title{
PET-CT Preparation: Solução Mobile na Preparação de Pacientes para o Exame 18-FDG PET CT
}

\author{
Celine Lacerda de Abreu Soares ${ }^{2}$, Fernando de Amorim Fernandes 2 , \\ Érito Marques de S. Filho ${ }^{2}$, Raffaele Giubbini ${ }^{3}$, Marina Gazzilli ${ }^{3}$, Hain Verberne, \\ Flávio Luiz Seixas ${ }^{2}$ e CláudioTinoco Mesquita ${ }^{1}$
}

${ }^{1}$ Cardiologia/ Departamento de Medicina Clínica - Faculdade de MedicinaUniversidade Federal Fluminense (UFF) Caixa Postal 24033-900 - Niterói/RJ - Brasil.

${ }^{2}$ Laboratório Midiacom - Escola de Engenharia Universidade Federal Fluminense (UFF) Caixa Postal 24210-240- Niterói/RJ - Brasil.

${ }^{3}$ Università degli Studi di Brescia - Brescia/BS -Itália.

${ }^{4}$ Amsterdam UMC Research Institutes - Meiberfdreef/AZ - Holanda.

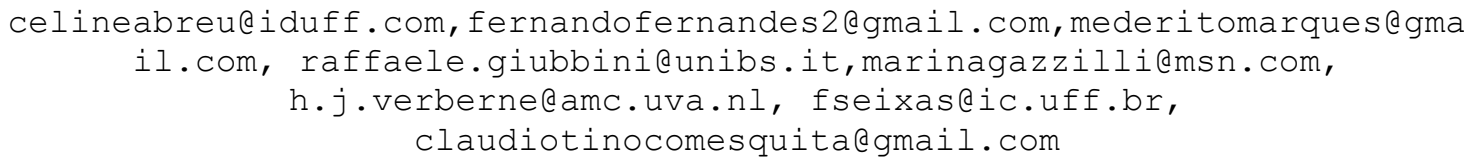

\begin{abstract}
The Positron Emission Tomography to cardiac examinations has been used as part of the image repertoire. A recurrent issue in the PEC-CT preparation is the necessity of reduction of the glucose capture by the myocardium. In order to achieve adequate suppression and, consequently, increased accuracy, it is mandatory to modify the diet three days before the $18 F-F D G C T$. Under the auspices of a multidisciplinary team, we are developing with the Ionic4 framework for multiplatform, a mobile application able to register and inform the user the proper diet that the patient should perform for three days before the exam. After being installed on smartphones, we will test the app for failures and security.
\end{abstract}

Resumo. A Tomografia por Emissão de Pósitrons para exames cardíacos vem sendo cada vez mais utilizada como parte do repertório de imagem. Um problema de rotina na preparação de PET-CT é a necessidade de redução da captação de glicose pelo miocárdio. Modificar a dieta três dias antes da $18 \mathrm{~F}$ FDG -CT é obrigatório para a adequada supressão e, consequentemente, um aumento da precisão. Sob os auspícios de uma equipe multidisciplinar, estamos desenvolvendo com o framework Ionic4 para multiplataforma, uma aplicação móvel capaz de registrar e informar ao usuário a dieta que o paciente deve realizar por três dias antes do exame. Depois de instalar em smartphones, o aplicativo deve ser testado quanto a falhas e segurança.

\section{Introdução}

A PET / CT com 18F-FDG é uma técnica valiosa de aquisição de imagem para o diagnóstico de endocardite infecciosa, capaz de oferecer um prognóstico claro sobre a patologia investigada. Recentemente, pesquisas apontaram um problema de rotina na preparação da PET-CT que é a necessidade de redução da captação de glicose pelo 
miocárdio (Bleeker-Rovers, 2017). Consequentemente, a modificação da dieta três dias antes da 18-FDG PET CT se apresenta como um fator de melhora expressiva quanto a precisão do exame, uma vez que beneficia a qualidade da imagem. Isso indica que o processo de vigilância vai além dos setores de medicina nuclear e requer a integração dos pacientes à medida em que estes devem se dedicar aos cuidados antecedentes à realização do exame. $\mathrm{O}$ objetivo do artigo é apresentar o estado atual do desenvolvimento de um aplicativo móvel capaz de fornecer melhorias na resolução de tarefas diárias, tendo em vista que smartphones e tablets são acessados por grande parte dos usuários dos sistemas de saúde. Assim, demonstraremos o funcionamento do aplicativo através da transição de telas dentro de um projeto de interface de usuário simulada.

\section{Trabalhos Relacionados}

Os trabalhos relacionados foram agrupados em dois subconjuntos. No primeiro subconjunto levantamos os aplicativos existentes para o acompanhamento nutricional (Subseção 2.1) e no segundo subconjunto buscamos funções complementares em outros aplicativos (Subseção 2.2).

\subsection{Aplicativos para Acompanhamento Nutricional}

Inicialmente foram escolhidos os aplicativos DSkids ${ }^{1}, \mathrm{MyFitnessPal}^{2}$, e WW Vigilantes ${ }^{3}$ do Peso, respectivamente. Para a avaliação, utilizou-se os seis critérios: (1) interface e usabilidade (2) base de alimentos, (3) elementos de motivação do usuário, (4) informações disponibilizadas ao usuário, (5) valor do aplicativo e (6) integração com o profissional responsável.

O aplicativo DSKids é um diário alimentar gratuito capaz de auxiliar os pais no acompanhamento das refeições de seus filhos. Sua interface possui caracterização infantil e oferece uma navegação prática sob uso de menu tradicional. No entanto, a base de alimentos é restrita e não há uma boa descrição do que foi consumido. Acrescenta-se o fato do software não prover opção de notificações para usuário nem via de comunicação com profissionais da área. Dessa forma, subentende-se que responsáveis especializados não conseguem acompanhar seus usuários por esta ferramenta.

O MyFitnessPal também é gratuito, mas seu pleno usufruto se restringe à categoria premium. Este aplicativo possui capacidade de registrar tanto refeições quanto exercícios físicos. Além disso, tem capacidade de escanear mais de 4 milhões de códigos de barras de alimentos dentre a sua base de dados estimada em 6 milhões. Isso garante uma inovação animadora para o usuário, que poupará tempo de pesquisa. Em relação ao design e disposição de elementos visuais o aplicativo deixa bastante a desejar, mas essa lacuna não interfere na usabilidade do produto. Por último, assim como o DSkids, não foi encontrado espaço de apoio de profissionais da área.

Já o WW Vigilantes do Peso é pago e restrito à usuários cadastrados. Diferentemente do MyFitnessPal, esse aplicativo possui layout organizado e rico em ilustrações, o que viabiliza uma navegação fácil. Elementos de gamificação também se

\footnotetext{
${ }^{1}$ http://www.dskids.com.br, acessado em Janeiro/2019

${ }^{2} \mathrm{https}: / / \mathrm{www} . \mathrm{myfitnesspal.com} / \mathrm{pt}$, acessado em Janeiro/2019

${ }^{3} \mathrm{https} / / \mathrm{www}$.vigilantesdopeso.com.br/br/aplicativo-vp, acessado em Janeiro/2019
} 
destacam neste software e se materializam em seu sistema de dieta baseada na contagem de pontos. Em relação ao banco de dados e registro, possui a qualidade de customização de alimento, mas isso não se sobrepõem às funcionalidades oferecidas pelo software mencionado anteriormente. Por fim, apesar de possuir botão para monitoramento de peso, o resultado apenas pode ser compartilhado através da comunicação pessoal -mostrandoo. Isso evidencia a falta de conexão da ferramenta mobile com um profissional especializado que possa acompanhar o usuário à distância.

\subsection{Aplicativos Acessórios}

Os aplicativos levantados anteriormente visavam registrar e acompanhar as refeições, mas não durante um ciclo ou período pré-determinado. Ademais, não possuíam informações importantes relacionadas à Medicina Nuclear. Esses motivos fomentaram o aumento do leque de pesquisa. Sendo assim, recorremos à softwares de acompanhamento de humor, menstrual e de preparo para exames de medicina nuclear, tais como Daylio ${ }^{4}$, $\mathrm{Flo}^{5}$ e PatientPreparationinNuclearMedicine ${ }^{6}$, respectivamente. Os critérios de análise nesta etapa foram: (1) interface e usabilidade, (2) elementos de motivação do usuário, (3) diferencial do produto em relação à primeira fase (4) informações acessíveis ao usuário, (5) valor do aplicativo e (6) integração com o profissional responsável.

Daylio é um diário de humor gratuito, mas só disponibiliza todos os recursos para usuário de categoria premium. Este aplicativo chama atenção pela simplicidade, boa disposição de conteúdo em seu menu tipo tabs na parte inferior da interface. Para fomentar a utilização, o software utiliza técnicas de gamificação. Assim, usuários ganham "prêmios" conforme aumenta a frequência de uso. Uma característica que diferencia este aplicativo dos demais levantados na primeira fase é a facilidade de exportar registros em documento csv para impressão, além de fazer back up e restauração via Google Drive. No entanto, não apresenta via de comunicação com profissionais da área abrangente.

Tanto quanto Daylio, Flo também faz uso do mesmo tipo de para navegação no software, mas supera o primeiro em relação ao design de sua interface. Ainda que seu propósito não tenha ligação à Medicina Nuclear ou alimentação, o aplicativo surge da ideia de acompanhamento de calendário - no caso menstrual - e cumpre muito bem esta proposta. Assim, possui a capacidade de registrar datas de início, fim e duração dos ciclos femininos além de promover os respectivos registros de cada dia. Entretanto, deixa a desejar em relação à exportação de dados e a comunicação com profissionais da área.

Por último e não menos importante, o PatientPreparationinNuclearMedicine é um aplicativo gratuito liberado pelo Inaya Medical College que não faz acompanhamento nas tarefas diárias, mas instrui os pacientes requisitados para o exame de Medicina Nuclear. Para isso, essa ferramenta disponibiliza informativos textuais sobre as etapas do exame, os medicamentos que devem ser avisados ou evitados e reações que devem ser observadas após o exame. O layout do aplicativo é o mais simples dentre os pesquisados e chama atenção por sua página inicial conter somente ícones. Ainda assim, seu uso é bastante

\footnotetext{
${ }^{4}$ https://daylio.webflow.io/, acessado em Janeiro/2019

${ }^{5} \mathrm{https} / / /$ flo.health/, acessado em Janeiro/2019

${ }^{6} \mathrm{https}: / /$ www.inaya.edu.sa/nmt.php, acessado em Janeiro/2019
} 
intuitivo. Não possui lembretes, elementos de motivação nem integração usuárioprofissional por se tratar de um software de caráter unicamente informativo.

Comparando os dois subconjuntos, evidenciou-se a possibilidade de combinação das características de um diário alimentar com o acompanhamento de calendários, disponibilização de informações médicas e interatividade. Assim, podemos desenvolver um produto de ideias customizadas com o diferencial de oferecer a ponte digital entre professional e usuário, ferramenta não disponível em nenhum software mobile pesquisado.

\section{Metodologia}

Um grupo multidisciplinar com profissionais de tecnologia e especialistas em medicina nuclear foi criado para desenvolver o aplicativo.

Discutiu-se a necessidade clínica e aprofundaram-se as pesquisas de soluções existentes para idealização do software. Em seguida, foi feita uma reunião com propósito de levantar os requisitos mínimos do sistema, registrá-los e, consequentemente, elaborar o diagrama de casos de uso, resultando a documentação da análise do sistema.

Para o desenvolvimento do sistema, escolheu-se o banco de dados Firebase e SDK Ionic 4 porque é multiplataforma e codificado com linguagem typescript, orientada a objetos. Isso permite maior facilidade de manutenção e escalabilidade do produto.

Essas definições possibilitaram construção do mockup, que é um modelo real de produto para a demonstração, visualização da interface e avaliação de design do que foi proposto inicialmente. Essa fase de trabalho exigiu intensa interação do grupo e trabalho coletivo, pois antes de levar a ideação para as ferramentas de apoio, cada tela do aplicativo foi desenvolvida manualmente pelos membros do grupo. Após transferência para plataforma digital sob o uso de Photoshop CS6, concluímos a transição das telas com a plataforma virtual de prototipação InVision? .

\section{Desenvolvimento do Aplicativo}

O cenário de uso do sistema PET-CT Preparation traduz-se nas etapas percorridas por um paciente na obtenção do histórico alimentar para a realização do exame PET-CT. Os atores que interagem com esse cenário são: paciente, profissional de saúde, médico. A fim de permitir o acesso simultâneo desses atores, desmembrou-se o sistema em duas partes: uma interface web administrativa e outra mobile para os pacientes. Para este trabalho foi confeccionado o alicerce da plataforma mobile: lista de requisitos, cenários de uso, produção de telas e prototipagem.

A primeira ordem de construção de projeto consistiu na listagem dos serviços fornecidos pelo sistema. Foi fundamental para reflexão das necessidades dos usuários. Para isso, foram consideradas suas qualidades específicas e restrições operacionais como

${ }^{7}$ https://www.invisionapp.com/ 
a necessidade de uma tela para registro de alimentos e que esse mesmo requisito funcione off-line.

Com essa listagem, foi possível obter a melhor compreensão da interação homemmáquina e consequentemente conceber a descrição e diagramação dos cenários de uso. Nessa conjuntura, segue a descrição UC1. O paciente foi encaminhado para fazer o exame e solicita o seu agendamento ao atendente do setor de Medicina Nuclear; UC2. O atendente cadastra o paciente e fornece um usuário e uma senha de acesso para liberar o uso do software mobile PET-CT PREPARATION; UC3. O paciente realiza o download do aplicativo; UC4. O paciente faz o login; UC5. O paciente concorda com os termos de uso (se existirem); UC6. O sistema alerta sobre a importância do uso do aplicativo e pergunta se pode iniciar seu funcionamento; UC7. O paciente autoriza; UC8. O sistema pergunta qual a data e horário de realização do exame; UC9. O paciente informa; UC10. O sistema o encaminha para a tela de contagem de tempo (ativo e de realização do exame); UC11. O paciente registra a suas refeições durante as $72 \mathrm{~h}$ antes do exame; UC12. O sistema envia um e-mail informando o histórico e confirmando se o preparo foi adequado antes de fazer o exame; UC13. Durante o laudo, o médico pode acessar o histórico alimentar do paciente via web.

Em sequência, a produção de telas e a prototipação puderam ser desenvolvidas sob as exigências feitas e orientadas à maior acessibilidade. Na próxima Seção será mostrado o protótipo desenvolvido e suas respectivas transições.

\section{Prototipação das Telas}

Para fazer uso dos recursos oferecidos pelo aplicativo, primeiramente, é necessário efetuar o login com a senha fornecida pelo professional de saúde (figura 3, tela 1). Após a autenticação dos dados, surge o menu principal (figura 3, tela 2) onde é possível acompanhar em qual etapa dos três dias o usuário se encontra e mostrar a data na qual o exame está marcado. Também há um botão (figura 3, tela 2) que permite o acréscimo direto de alimentos naquele dia e que encaminha o usuário para a página de diário alimentar.

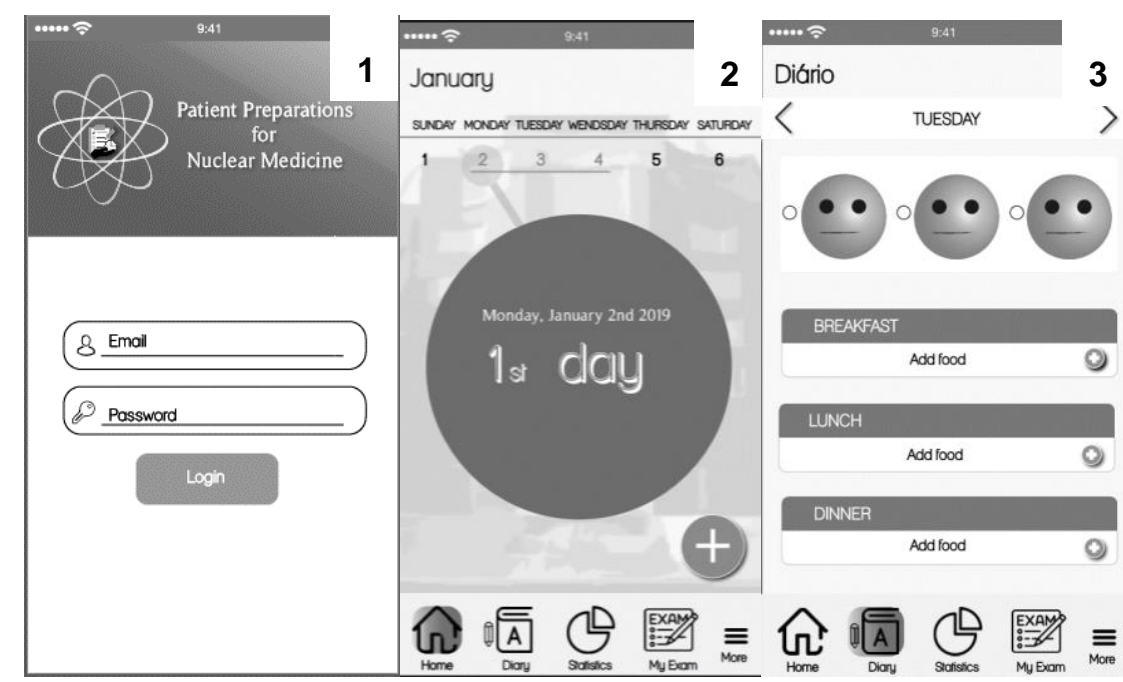

Figura 3. Protótipo do sistema PET-CT Preparation: telas de login (1), Início (2) e diário (3). 
Para transitar entre as telas estabelecidas e dar sequência ao acompanhamento diário, exibe-se um menu horizontal na parte inferior da tela do aplicativo que permite acessar além da tela inicial o diário alimentar (figura 3, tela 3), estatísticas e indicações (figura 4, tela 4) e uma página com todas as informações descritivas daquele exame (figura 4, tela 5). Outras ferramentas estão em estudo e estarão disponíveis em "more" (figura 4, tela 6). Desta forma o software é capaz de oferecer ao usuário apoio, diretriz e esclarecimento.

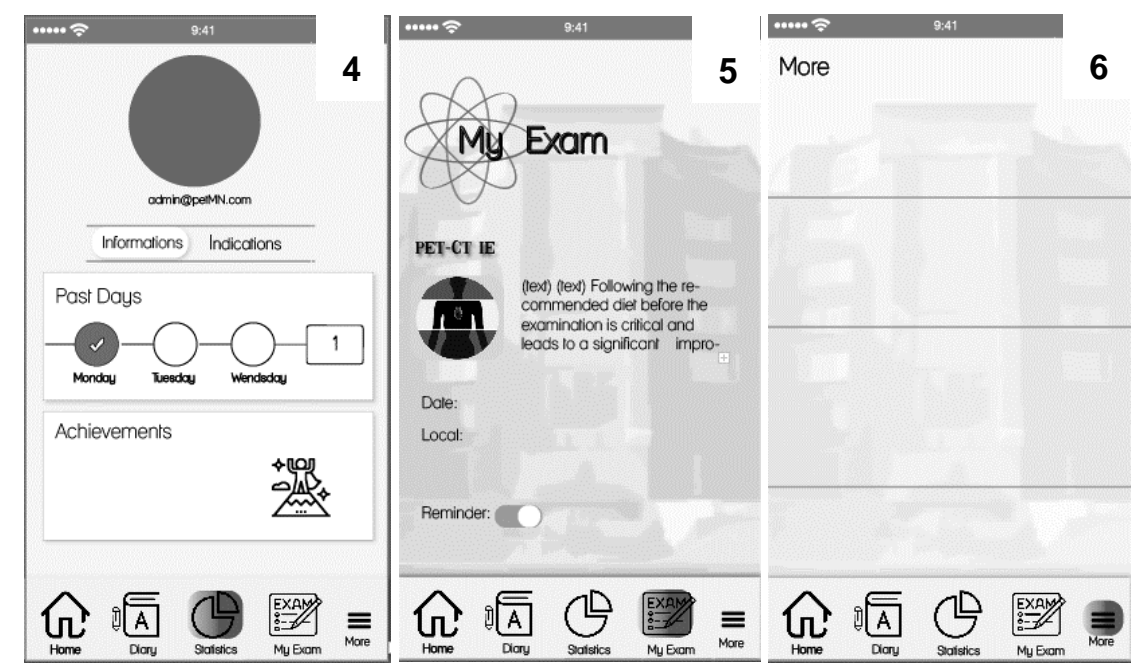

Figura 4. Protótipo do sistema PET-CT Preparation: telas de estatísticas (1), exame (2) e menu lateral (3).

\section{Conclusão e Considerações Finais}

O aplicativo PET-CT Preparation consiste numa ferramenta de apoio a tarefas diárias, acompanhamento médico e também de educação ao paciente, pois oferece informações e esclarecimentos que muitas vezes não conseguem ser passadas no momento de atendimento. Diante disso, durante o desenvolvimento inicial foi possível construir um protótipo e testar se os requisitos foram considerados quando o usuário interagir com $o$ projeto. E assim, estimar a complexidade do projeto, analisar com mais profundidade a questão da usabilidade e design e garantir maior eficiência no desenvolvimento do sistema.

Em relação aos próximos passos, a implementação de software móvel está ocorrendo agora e tem o potencial de tornar as rotinas clínicas mais práticas e acessíveis para a equipe do hospital e pacientes. O aplicativo deverá ser capaz de informar, apoiar, confortar os usuários, economizar tempo na explicação do procedimento PET CT e nas questões críticas na preparação do paciente. A adoção dessa tecnologia poderá evitar a repetição do exame (o que ocorre quando o preparo não é bem feito), que consequentemente reduz a exposição dos pacientes a radiação, o desgaste de realizar um novo procedimento e o custo para o Serviço de Medicina Nuclear (ampliando vagas no caso do SUS), além de oferecer praticidade, democratização do acesso e fornecer indicadores de qualidade para o departamento.

\section{Referências}

Bleeker-Rovers, CP. (2017) "The Value of 18F-FDG PET/CT in Diagnosis and During Follow-up in 273 Patients with Chronic Q Fever" http://jnm.snmjournals.org/cgi/pmidlookup?view=long\&pmid=28546336 
DSKIDS. Versão 3.1.4.0 Under Armour, 2015. Disponível em: $<$ http://www.dskids.com.br/>. Acesso em 12 de dezembro de 2018.

DAYLIO. Versão 1.20.1 Under Armour, 2015. Disponível em: $<$ https://daylio.webflow.io/>. Acesso em 13 de fevereiro de 2019.

FLO. Versão 4.17.2 Under Armour, 2016. Disponível em: $<$ https://flo.health/>. Acesso em 1 de fevereiro de 2019.

MYFITNESSPAL. Versão 19.1.5 Under Armour, 2019. Disponível em: $<$ https://www.myfitnesspal.com/pt $>$. Acesso em 4 de fevereiro de 2019.

PATIENTPREPARATIONINNUCLEARMEDICINE. Versão 2.0.0 Under Armour, 2018. Disponível em: < https://www.inaya.edu.sa/nmt.php>. Acesso em 12 de dezembro de 2019.

WWVIGILANTESDOPESO. Versão 7.6.0 Under Armour, 2017. Disponível em: $<$ https://www.vigilantesdopeso.com.br/br/aplicativo-vp $>$. Acesso em 18 de janeiro de 2019. 